\title{
A Humanoid Robot Gait Planning and Its Stability Validation
}

\author{
Jian Zeng, Haibo Chen, Yan Yin \\ Department of Modern Mechanics, University of Science and Technology of China, Hefei, China \\ Email: eyuren@mail.ustc.edu.cn, hbchen@ustc.edu.cn, yinyan@mail.ustc.edu.cn
}

Received August 2014

\begin{abstract}
Gait planning based on linear inverted pendulum (LIPM) on structured road surface can be quickly generated because of the simple model and definite physical meaning. However, over-simplification of the model and discontents of zero velocity and acceleration boundary conditions when robot starts and stops walking lead to obvious difference between the model and the real robot. In this paper, parameterized gait is planned and trajectories' smoothness of each joint angle and centroid are ensured using the 3-D LIPM theory. Static walking method is used to satisfy zero velocity and acceleration boundary conditions. Besides, a multi-link model is built to validate the stability. Simulation experiments show that: despite of some deviation from the theoretical solution, the actual zero-moment point (ZMP) is still within the support polygon, and the robot walks steadily. In consequence, the rationality and validity of model simplification of LIPM is demonstrated.
\end{abstract}

\section{Keywords}

Humanoid Robot, Gait Planning, 3-D LIPM, Stability Validation, Multi-Link Model

\section{Introduction}

Reasonable walking pattern planning is the foundation to achieve stable bipedal walking due to the complexity of a humanoid robot mechanism. So far the mainstream gait planning methods are based on the simplified physical model [1], the bionics [2], the energy optimal method [3], the intelligence technology [4] et al. The first method has a simple model and definite physical meaning, but the robustness, Anti-jamming ability and adaptability to the practical walking environment are poor due to the oversimplification from the real model. The database does not have the feature for sharing due to the difference between the robot and the human for the second method. The gait planning method based on energy optimization can not be used to real-time application for its complexity in modeling and optimization procedure. The intelligent algorithm was proposed in recent years and there are a series of problems remained to be solved, including the algorithm's effectiveness, real-time performance, convergence et al. [5]. This paper is confined to the robot walking on flat ground, which is simple but the robot might lose its stability if an unsuitable walking pattern and gait parameters are selected. The 3-D LIPM is used for gait planning, thus leading to simple formula, definite physical meaning and outstanding adaptability to structured roads. 
The ultimate aim of gait planning is to realize a stable walking, so walking stability needed to be checked to confirm the feasibility and effectiveness of the gait planning. The model of 3-D LIPM has deviations with real robot on its assumption that the mass of the robot is condensed to one point and the mass of lower limbs is neglected [6], which will usually lend to instability. So in this paper, walking stability based on the ZMP stability criterion is calculated by using a multi-link model which is closer to the real robot by considering the lower limbs' mass. Meanwhile the virtual prototype is built for simulation. Through these two treatments, the rationality and validity of the simplification of the 3-D LIPM is verified.

\section{Humanoid Robot Kinematics Modeling}

The robot can be simplified into a multi-link model of 12 degrees of freedom assuming that the upper body keeps upright and relative rest when it walks. The local coordinate system at each joint is set up as Figure $\mathbf{1}$ by D-H rule [7]. The 0 coordination is the world coordinate system (WCS) fixed on the ground. 1 - 12 coordinations are the local coordinations. After calculating the homogeneous transformation matrixes between the local coordinations, and knowing the angles at each joint, the WCS coordinates of any point in the local coordinate system can be calculated based on the chain rule of homogeneous transformation, thus providing the WCS coordinates and orientations of each link for stability validation.

\section{Humanoid Robot Gait Planning}

Each joint angle trajectory can be calculated by inverse kinematics once the trajectories of the center of mass (CM) and the swing legs are planned, thus the corresponding gaits are generated. The trajectories of swing legs are fitted by polynomial interpolation through the parameters as step length, step cycle, zero speed and zero acceleration at the time swing leg leaving and landing ground. Here we focus on the design of trajectory for CM.

\subsection{Trajectory of CM}

Figure 2 shows the 3-D LIPM which is proposed for trajectory planning of CM under the assumption that the mass of lower limbs is neglected, the mass of the upper body is condensed to one point and its vertical height $Z_{c}$ remains constant.

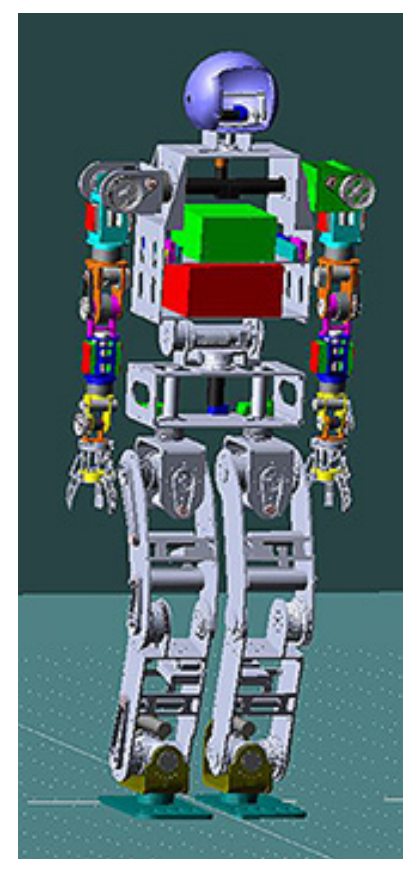

(a)

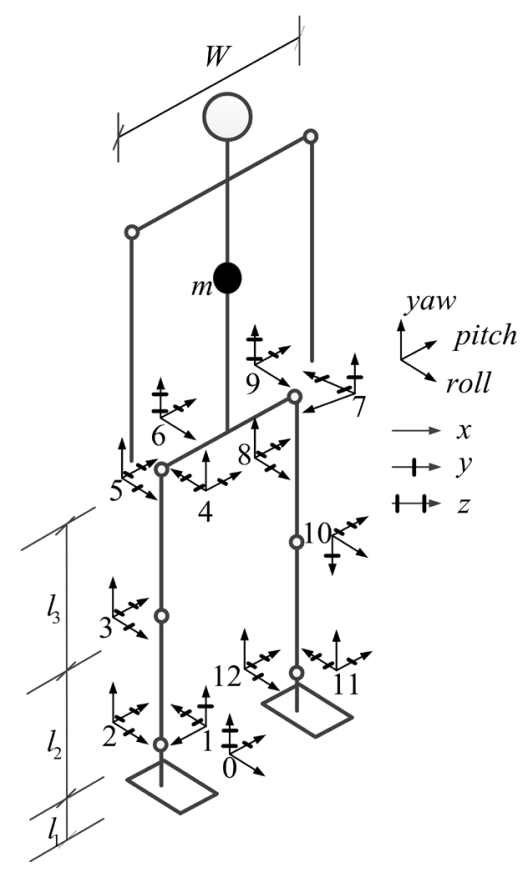

(b)

Figure 1. Virtual prototype and multi-link model: (a) Virtual prototype model; (b) Multi-link model. 

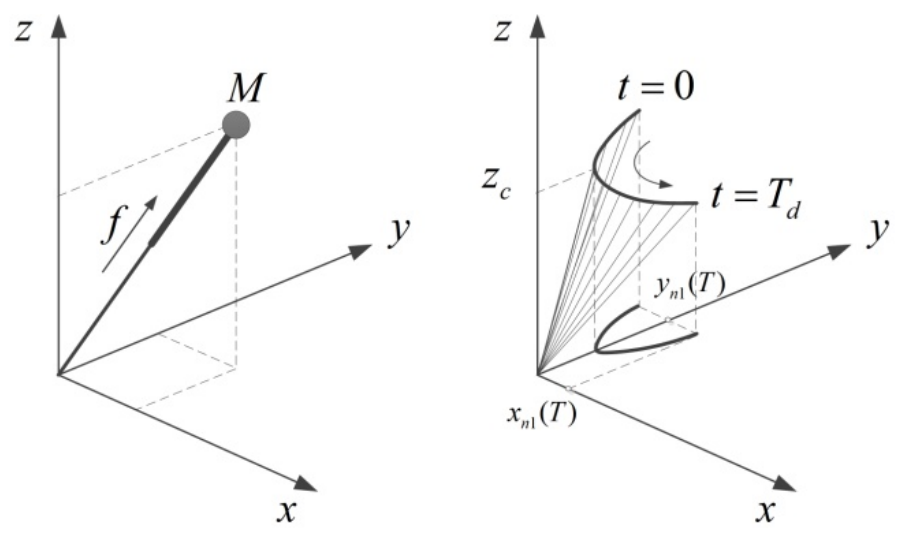

Figure 2. 3-D LIPM model and its trajectory.

In this case, the motion differential equations of 3-D LIPM are given by:

$$
\left\{\begin{array}{l}
\ddot{x}=\frac{g}{z_{c}} x \\
\ddot{y}=\frac{g}{z_{c}} y
\end{array}\right.
$$

The solutions are:

$$
\left\{\begin{array}{l}
x(t)=x(0) \cosh \left(t / T_{c}\right)+T_{c} \dot{x}(0) \sinh \left(t / T_{c}\right) \\
y(t)=y(0) \cosh \left(t / T_{c}\right)+T_{c} \dot{y}(0) \sinh \left(t / T_{c}\right)
\end{array}\right.
$$

And the first derivatives versus time are:

$$
\left\{\begin{array}{l}
\dot{x}(t)=x(0) / T_{c} \sinh \left(t / T_{c}\right)+\dot{x}(0) \cosh \left(t / T_{c}\right) \\
\dot{y}(t)=y(0) / T_{c} \sinh \left(t / T_{c}\right)+\dot{y}(0) \cosh \left(t / T_{c}\right)
\end{array}\right.
$$

where, $T_{c}=\sqrt{z_{c} / g}$.

\subsubsection{Middle Gait}

For single leg support phase: assuming the time section is $[0, T]$, the CM trajectory component for $x$ vector and $y$ vector are $x_{n 1}(t)$ and $y_{n 1}(t)$, respectively. Because of symmetry and periodicity conditions, it is required that:

$$
\left\{\begin{array}{l}
x_{n 1}(T)=-x_{n 1}(0), \dot{x}_{n 1}(T)=\dot{x}_{n 1}(0) \\
y_{n 1}(T)=y_{n 1}(0), \dot{y}_{n 1}(T)=-\dot{y}_{n 1}(0)
\end{array}\right.
$$

Substituting Equation (4) into Equation (3), one gets

$$
\left\{\begin{array}{l}
\dot{x}_{n 1}(0)=\frac{x_{n 1}(0)\left(1+e^{\frac{T}{T_{c}}}\right)}{T_{c}\left(1-e^{\frac{T}{T_{c}}}\right)} \\
\dot{y}_{n 1}(0)=\frac{y_{n 1}(0)\left(1-e^{\frac{T}{T_{c}}}\right)}{T_{c}\left(1+e^{\frac{T}{T_{c}}}\right)}
\end{array}\right.
$$


When the initial CM positions $x_{n 1}(0)$ and $y_{n 1}(0)$ are given, the trajectory of CM can be calculated by substituting Equation (5) into Equation (2).

For double leg support phase, in order to keep the velocity and acceleration of CM continuous in the period that the robot walks from one final LIPM moment to the next initial LIPM moment, a double leg support phase needs to be considered. The sketch of $x$ axis is shown in Figure 3.

Assuming the time section is $\left[T, T_{d}\right]$, the $x$ and $y$ vector trajectories of CM $x_{n 2}(t)$ and $y_{n 2}(t)$ can be calculated by piecewise polynomial interpolation.

\subsubsection{Start Gait and Final Gait}

The start gait and final gait in single leg support phase correspond to the period of $\left[\frac{T}{2}, T\right]$ and $\left[0, \frac{T}{2}\right]$, respectively. The CM trajectories of this phase can be calculated in the same way by considering the symmetry and periodicity conditions.

The robot keeps static before starting to walk. To satisfy the continuous requirements of velocity and acceleration of $\mathrm{CM}$, the swing legs need to retreat to get an accelerating process for CM until the velocities and accelerations are equal to the initial start time of LIPM, assuming the section time is $\left[0, T_{s}\right]$. Similarly, after the final gait, the swing legs need to take a distance forward from support leg, and the CM moves from the final state of LIPM to static in the double leg support phase. The trajectories of above static walking phases are fitted by polynomial interpolation.

\subsection{Joint Angle Trajectory}

It is supposed that $T=1.0 \mathrm{~s}, T_{d}=1.2 \mathrm{~s}, T_{\mathrm{s}}=2.5 \mathrm{~s}, \mathrm{~s}_{1}=0.35 \mathrm{~m}, \mathrm{~s}_{2}=0.4 \mathrm{~m}, w=0.08 \mathrm{~m}, \mathrm{z}_{\mathrm{c}}=0.9 \mathrm{~m}$. The trajectories of lower limb joint angles including the phase $\left[0, T_{s}\right]$, start gait by left leg, three cycles of middle gait, and the final gait are calculated by inverse kinematics as Figure 4 (take the right leg for example).

\section{Stability Validation}

\subsection{Theoretical Calculation}

A multi-link model, shown in Figure 1(b), is used for theoretical calculation. It is assumed that inertial tensor of each link around its CM is neglected, the equations of ZMP are as follows:

$$
\begin{aligned}
& p_{x}=\frac{\sum_{i=1}^{N} m_{i}\left\{\left(\ddot{z}_{i}+g\right) x_{i}-\left(z_{i}-p_{z}\right) \ddot{x}_{i}\right\}}{\sum_{i=1}^{N} m_{i}\left(\ddot{z}_{i}+g\right)} \\
& p_{y}=\frac{\sum_{i=1}^{N} m_{i}\left\{\left(\ddot{z}_{i}+g\right) y_{i}-\left(z_{i}-p_{z}\right) \ddot{y}_{i}\right\}}{\sum_{i=1}^{N} m_{i}\left(\ddot{z}_{i}+g\right)}
\end{aligned}
$$

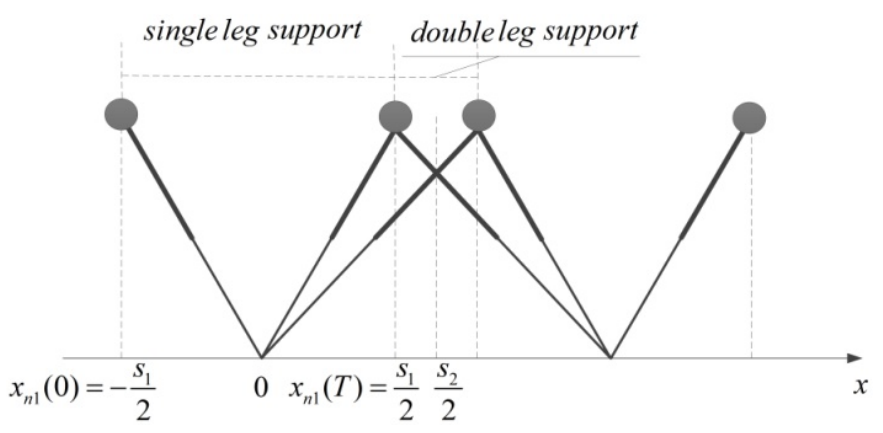

Figure 3. A Complete gait period on $x$ axis. 

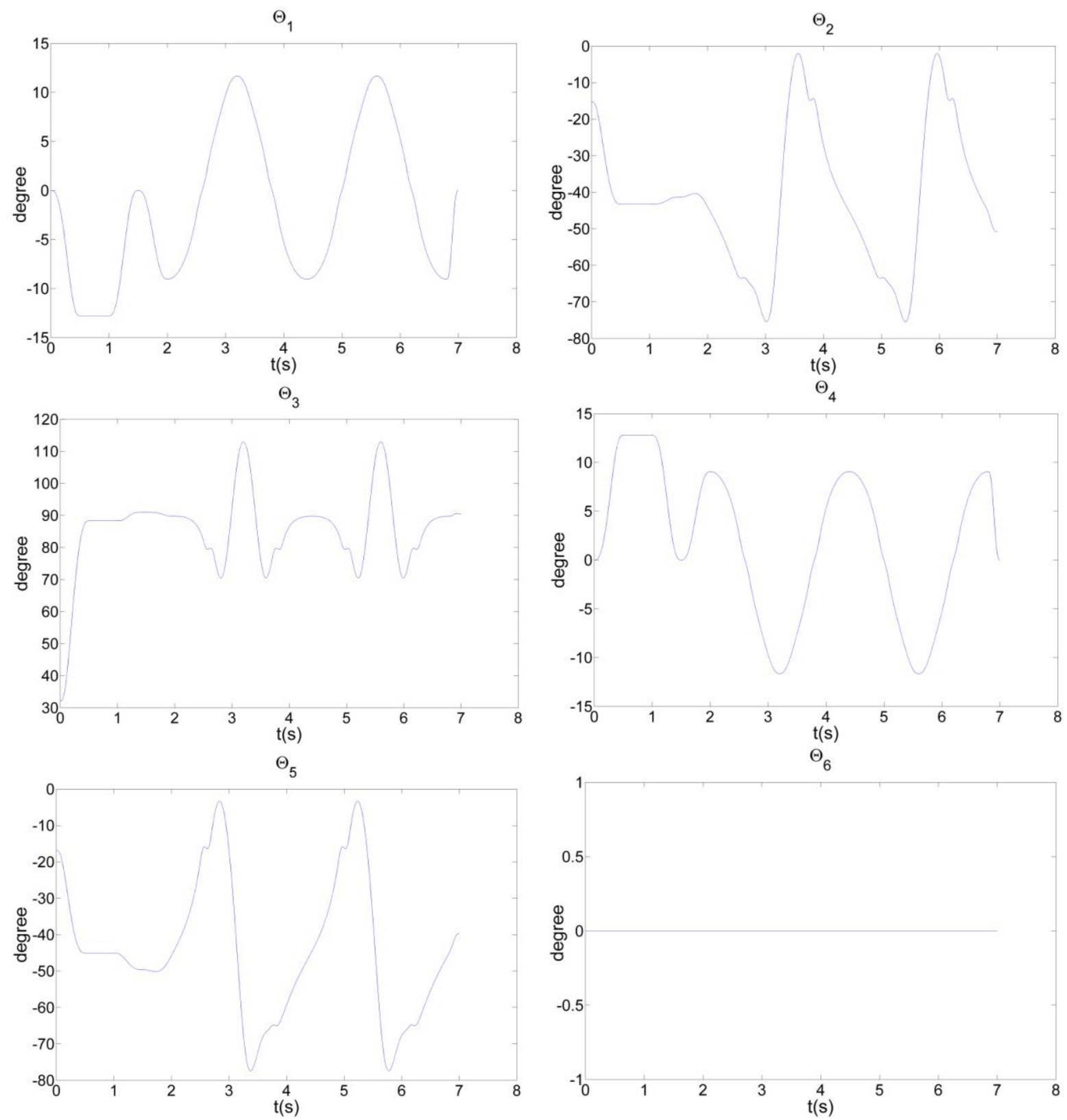

Figure 4. Joint angle trajectories of right leg.

The WCS trajectories of CM of each link are calculated by substituting each joint angle from the gait planning into multi-link kinematics model, where the victory and acceleration are calculated by the difference method. Substituting all these above into Equations (6) and (7), the solutions of ZMP trajectories are shown in Figure 5.

It is indicated by comparing Figure 5(a) and Figure 5(b) that walking stability improves obviously in the static walking phase $\left[0, T_{s}\right]$ on the start and stop moment and double leg support phase when two gait switching gets smooth and conforms to the actual rule. Figure 5(b) shows that the actual ZMP trajectory $p_{y}$ has deviation from the theoretical value. However, after the two treatments are introduced in, the actual ZMP is within the support polygon which means the deviation is very small, especially in single leg support phase, the stability margin is enough for steadily walking. By the way, the CM trajectories in double leg support phase need to continuously optimized. Thus it is theoretically proved that the differences do exist between the 3-D LIPM and the actual robot, but the simplification is reasonable and effective. 


\subsection{Virtual Prototype Simulation}

Solidworks is used to build the virtual prototype of a humanoid robot, ADAMS is used to simulate the robot's walking, the simulation diagram of one of the cycle gait is shown in Figure 6.

With the simulation result, it is concluded that the robot can walk steadily according to the planned gait. The rationality and effectiveness of 3-D LIPM is further confirmed by simulation.

\section{Conclusions}

LIPM is a common model for humanoid robot gait planning, but the model is over-simplified, ignoring the mass of lower limbs, the moment of inertia et al., which lead to obvious deviation from the real robot. Based on LIPM gait planning, a multi-link model is used to calculate the ZMP for stability validation. At the same time, a virtual
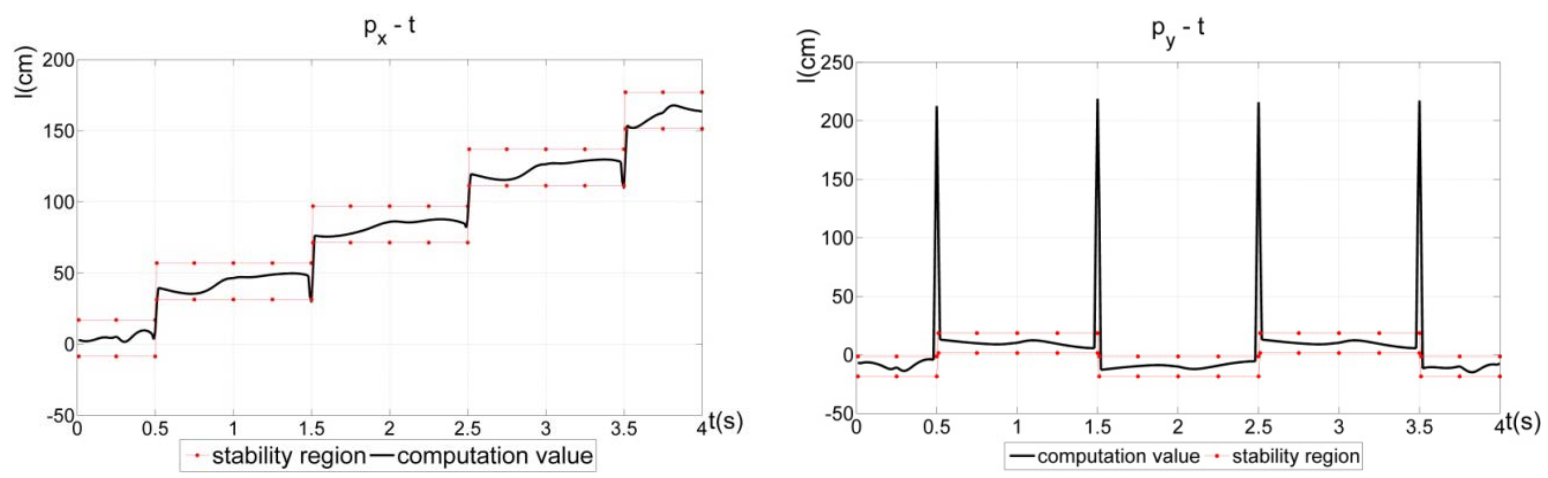

(a)
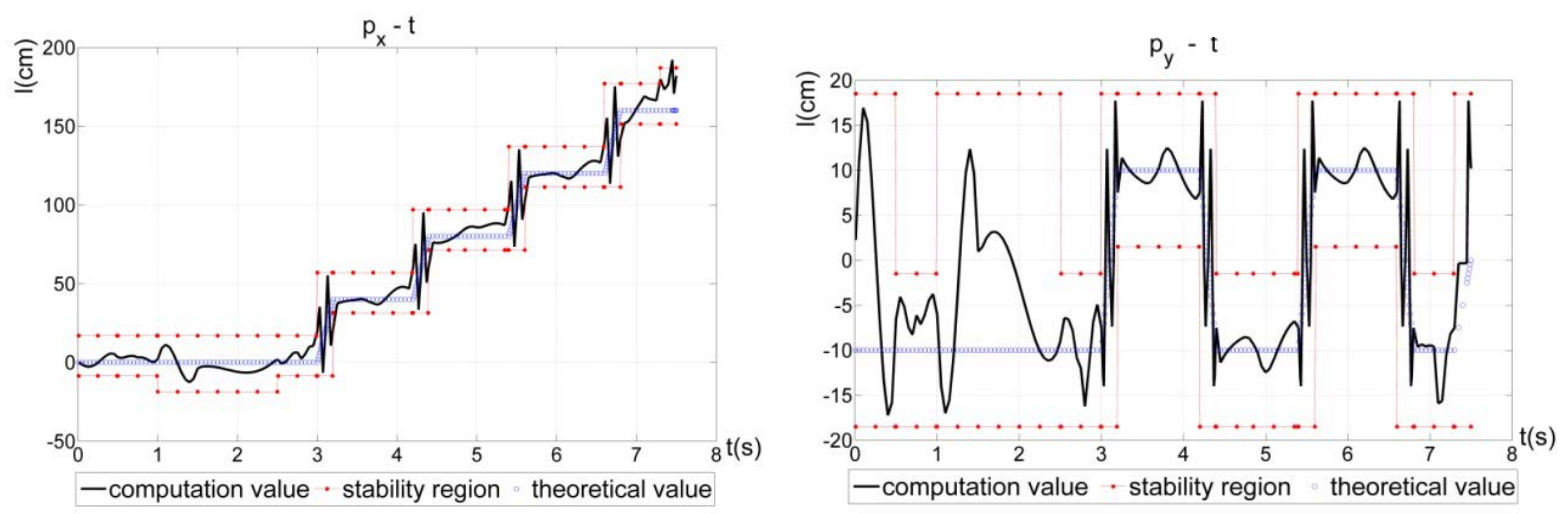

(b)

Figure 5. Trajectory of ZMP: (a) Trajectory of ZMP without static walking phase and double leg support phase; (b) Trajectory of ZMP with static walking phase and double leg support phase.
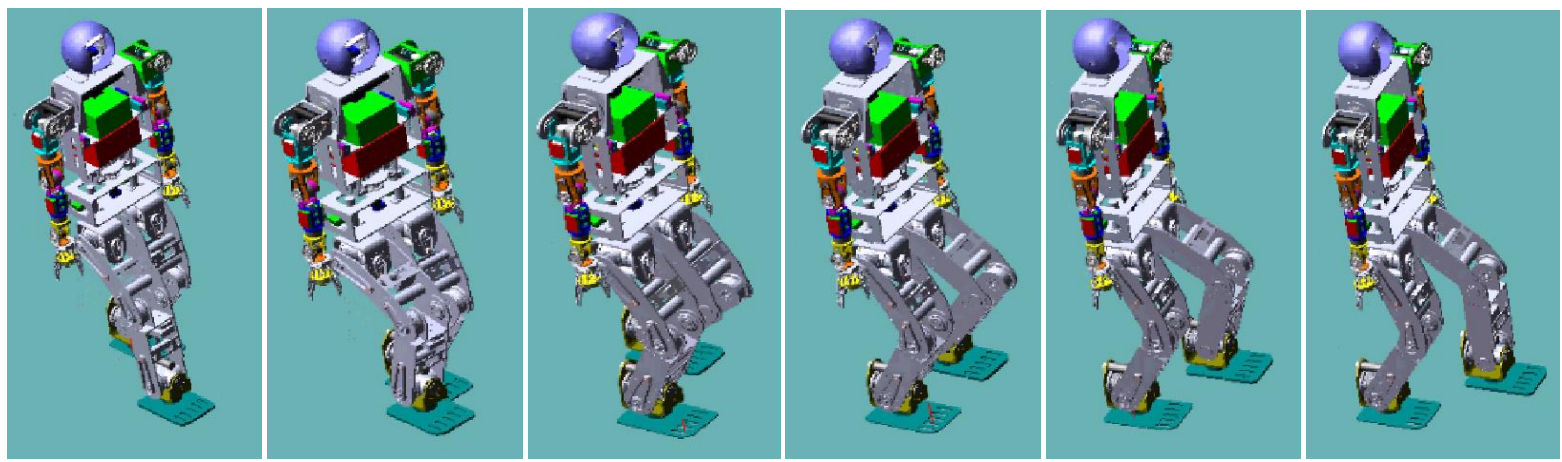

Figure 6. Virtual prototype simulation result. 
prototype is built for simulation. By gait planning and stability validation above, conclusions are drawn as follows:

1) 3-D LIPM is effective for structured pavement gait planning, the additional improvement of boundary conditions on the start and stop moment is effective and conforms to the actual rule.

2) The trajectories of joint angles are designed more smoothly after considering the speed and acceleration discontinuity problems when two gait cycle switching, which increases the walking stability of the robot.

3) The effect on ZMP by LIPM simplification is shown and the rationality of simplification is verified by theoretical calculation and simulation. Thus, the simplification can be used for real robot walking planning.

4) The stability can be validated by the relationship between ZMP trajectory and support polygon when robot walks, which is applicable to most structured road conditions.

\section{References}

[1] Fu, C.L. and Chen, K. (2008) Gait Synthesis and Sensory Control of Stair Climbing for a Humanoid Robot. IEEE Transactions on Industrial Electronics, 55, 2111-2120. http://dx.doi.org/10.1109/TIE.2008.921205

[2] Hirai, K., Hirose, M., Haikawa, Y. and Takenaka, T. (1998) The Development of Honda Humanoid Robot. Proceedings of the 1998 IEEE International Conference on Robotics and Automation, Leuven, 16-20 May 1998, 1321-1326.

[3] Nguyen, T.P., Kim, D.W., Kim, H.K. and Kim, S.B. (2008) An Optimal Control Method for Biped Robot with Stable Walking Gait. Proceedings of the 2008 IEEE-RAS International Conference on Humanoid Robots, Korea, 1-3 December 2008, 211-218.

[4] Katic, D. and Vukobratovic, M. (2003) Survey of Intelligence Control Techniques for Humanoid Robots. Journal of Intelligence and Robotic Systems, 37, 117-141. http://dx.doi.org/10.1023/A:1024172417914

[5] Fu, G.P. and Yang, Y.M. (2011) Survey and Prospect on Walking Control Strategies for Humanoid Robot. Machine Tool \& Hydraulics, 39, 154-158. (in Chinese)

[6] Shuuji, K., Hirohisa, H., Kazuhito, Y. and Kensuke, H. (2005) Humanoid Robots. Ohm-sha Ltd, Tokyo.

[7] Chen, K. and Fu, C.L. (2010) Humanoid Robot Theory and Technology. Tsinghua University Ltd., Beijing. (in Chinese) 